\title{
EFECTOS ECONÓMICOS DE LA CONSTRUCCIÓN DE LA VIVIENDA EN EL ECUADOR
}

\author{
ROLAND ZISS \\ El Colegio de México
}

EL ARTículo presenta un método para analizar cuantitativamente los efectos económicos de la construcción de viviendas sobre el empleo, los ingresos y la importación, tomando en cuenta la producción y distribución de los materiales de construcción. Además, ofrece los resultados de una investigación empírica de tres tipos de vivienda en Quito, Ecuador: uno, llevado a cabo bajo el auspicio de un programa estatal de interés social, considerado como vivienda formal, y otros dos consistentes en mo dificaciones de vivienda y casas construidas por auto-ayuda denominado irformal.

Los efectos económicos se componen de una parte directa que se produce en la misma obra, y una parte indirecta a través del uso de los materiales. Comparando los efectos sobre la ocupación y los ingresos en los diferentes tipos de vivienda, se llega a la conclusión que con informalidad creciente en la construcción de la vivienda se incrementan estos efectos, dada una suma constante de costos totales de construcción; aquéllos disminuyen para la realización de una superficie techada de igual dimensión y finalmente, se da un aumento de la proporción de los efectos indirectos. Esto indica que la construcción informal está interrelacionada más intensamente con los sectores preliminares de los materiales, que la construcción formal de vivienda.

Los contenidos de importación son casi iguales en los tipos de vivienda estudiadas y representan casi la mitad de los costos de materiales o casi un tercio de los costos totales de construcción. Para el diseño de una política nacional de vivienda valdría la pena tomar en cuenta los efectos analizados. A los planificadores de cualquier tipo de vivienda se les ofrece además, un método para comparar efectos económicos cuando el uso alternativo de materiales de construcción sea posible.

\section{Notas preliminares metodológicas}

1.1. Los efectos analizados: efecto de ocupación, efecto de ingresos y contenido de importación

\subsubsection{Efecto de ocupación}

Se entiende por "efecto de ocupación de la construcción de vivienda", a la cantidad de trabajo necesaria para la realización de espacio habita- 
ble; éste se mide en magnitudes físicas (como por ejemplo en días-hombre). Podemos distinguir un efecto de ocupación directo, que estaría dado por la cantidad de trabajo necesario para la producción directa de espacio habitable como parte del proceso constructivo de la obra, y uno indirecto, que está incorporado en los materiales de construcción necesarios para la construcción de vivienda, y se define como la cantidad de trabajo necesario para la producción y distribución de estos materiales de construcción. Se considerarán solamente las cantidades de trabajo utilizados directamente en la producción y distribución de materiales de construcción. La ocupación contenida en los productos intermedios y en las maquinarias necesarias para la producción de materiales de construcción, no será analizada, al igual que la ocupación contenida en la realización de otras actividades preliminares de la construcción de vivienda, como por ejemplo la planificación.

\subsubsection{Efecto de ingresos}

Se entiende por "efecto de ingresos de la construcción de vivienda", al ingreso que se produce en la realización de espacio habitable. Se mide en unidades monetarias, y representa la correspondencia monetaria con el efecto de ocupación. También hay un efecto de ingreso directo, que se genera por la producción directa de espacio habitable como parte del proceso constructivo de la obra, y uno indirecto, generado por la producción y distribución de materiales de construcción. Para el efecto de ingreso indirecto, sólo se consideran los ingresos obtenidos directamente en la producción y distribución de materiales de construcción. No se tendrán en cuenta los ingresos provenientes de sectores anteriores a la producción y distribución de materiales de construcción, como por ejemplo, los de productos intermedios, maquinarias para la producción de materiales de construcción o medios de transporte, al igual que los producidos en la realización de otras actividades preliminares a la construcción de vivienda.

\subsubsection{Contenido de importación}

El "contenido de importación de la construcción de vivienda", es el resultado de la introducción de rendimientos preliminares extranjeros, en la realización de espacio habitable. En la construcción de viviendas para estratos de bajos ingresos, por regla general, no participan fuerzas de trabajo extranjeras. En caso de utilizarse en la obra maquinarias de construcción importadas, tampoco serán consideradas para el cálculo del contenido de importación. El contenido de importación en la construcción de vivienda está incorporado, por lo tanto, solamente en los materiales de construcción utilizados. Se trata de una magnitud monetaria y tiene que ser relacionada con otros valores (como por ejemplo los costos de los 
materiales de construcción, los costos totales de construcción). El contenido de importación de la construcción de vivienda, indica la parte del valor que corresponde a importaciones del extranjero. Puede tratarse tanto de una importación directa de materiales de construcción, como de importación de medios de producción, de productos intermedios, y de medios de transporte para la producción y distribución nacional de materiales de construcción.

Mientras que los efectos de ocupación y de ingresos representan productos nacionales, el contenido de importación representa las relaciones con el comercio exterior, o sea, la dependencia con el exterior en la construcción.

\subsection{Modo de operación}

El modo de operar para obtener los efectos de ocupación, efectos de ingresos y contenidos de importación en la construcción de vivienda, está representado esquemáticamente en el cuadro 1.

Los efectos de ocupación y de ingresos directos, se obtienen con ayuda de una investigación cuantitativa de los participantes del proceso constructivo con relación al trabajo realizado y a los ingresos que debido a este trabajo se producen.

Para la obtención de los efectos de ingresos y de ocupación indirectos y de los contenidos de importación, se indagan los flujos físicos de los materiales de construcción que llegan a la construcción de vivienda, desde el comienzo hasta la finalización.

Los efectos de ocupación y de ingresos totales se logran resumiendo los efectos directos e indirectos.

\subsection{Tipos de la vivienda popular y dimensiones de referencia}

Efectos de ocupación, efectos de ingresos y contenidos de importación fueron estudiados en tres tipos de construcción de vivienda encontradas en dos urbanizaciones de hogares de bajos ingresos en Quito:

- El programa estatal de construcción de vivienda de interés social en MENA I (formal),

- las ampliaciones y modificaciones individuales en MENA I (informal),

- la construcción de vivienda por auto-ayuda organizada por el Comité del pueblo en ELof́sa (informal).

Para los tres tipos de construcción de vivienda, se utilizaron los datos obtenidos a nivel de las urbanizaciones. En el caso del programa de construcción de vivienda estatal de MENA I, los datos están basados en las 
Esquema 1

ESQUEMA DEL MODO DE OPERACIÓN PARA LA OBTENCIÓN DE LOS EFECTOS DE OCUPACIÓN, EFECTOS DE INGRESOS Y CONTENIDOS DE IMPORTACIÓN EN LA CONSTRUCCIÓN DE VIVIENDA

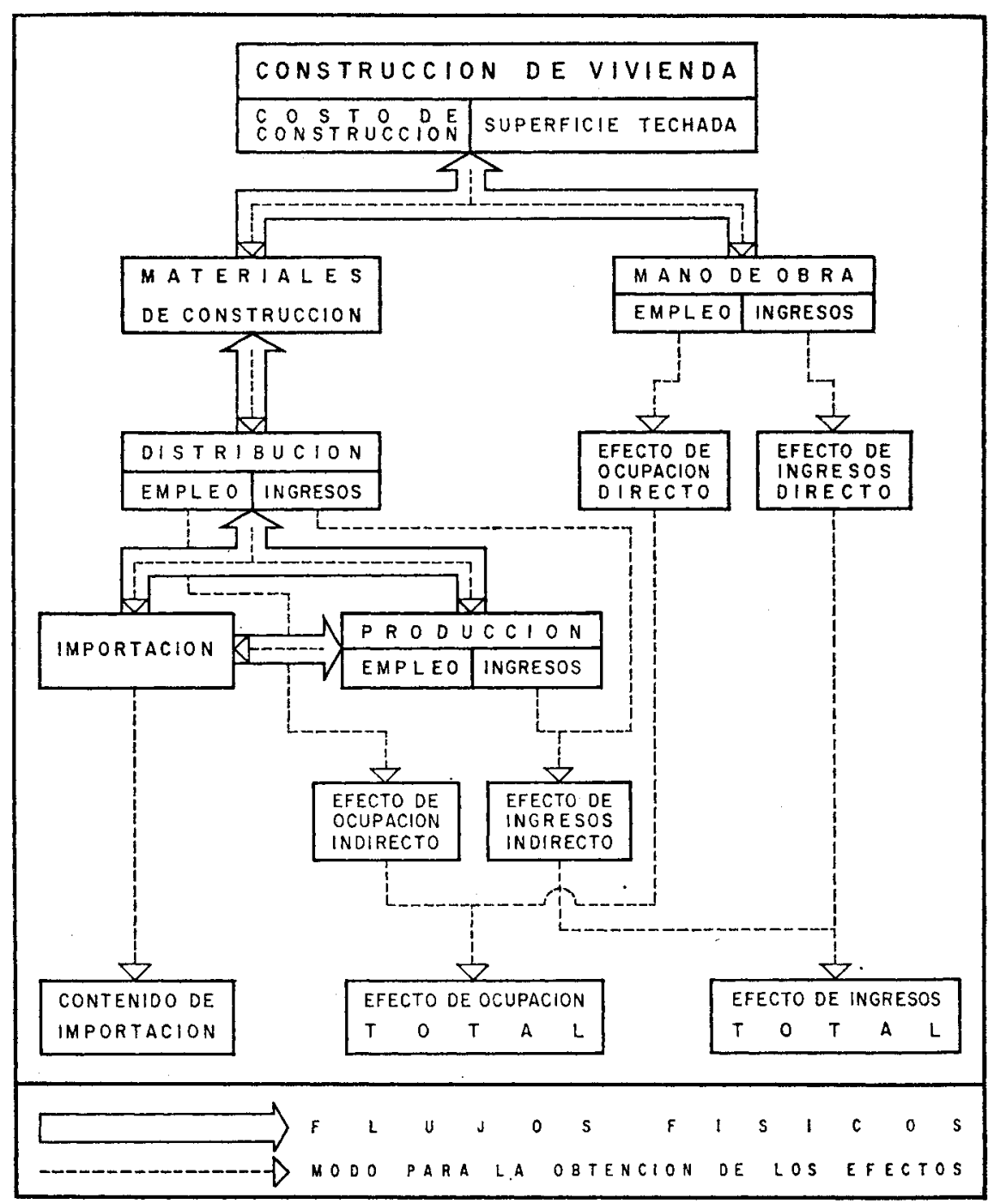


informaciones de la Junta Nacional de la Vivienda sobre un total de 1082 unidades construidas. La base informativa para los efectos de las actividades de construcción informal, surge de relevamientos propios, consistentes en 27 entrevistas con propietarios constructores en MENA I y de 26 en ELoísA. Utilizando datos agregados, se obtuvieron efectos promedio característicos para los diferentes tipos de construcción.

Para poder comparar los efectos de diferentes tipos de construcción de vivienda, éstos están referidos a una unidad básica: una cantidad física o una monetaria. Como magnitud de referencia física, se ha escogido un metro cuadrado de superficie techada; como medida de referencia monetaria, lo construido con un costo de $\$ 1000.00$ en $1977 / 78$.

\subsection{La situación en el mercado de los materiales de construcción}

Con el incremento de la extracción de petróleo, desde 1973, comienza un auge de la construcción sin antecedentes en la historia del Ecuador, con consecuencias decisivas para la disponibilidad y los precios de los materiales para la construcción de vivienda. La construcción de edificios comerciales y públicos, y también las obras de infraestructura, tienen una alta demanda de materiales que también se usan en las viviendas; esto provoca una reducción general de la oferta.

No todos los materiales de construcción tienen, en la misma proporción, la posibilidad de ser utilizados alternativamente, y de modo concurrente, en los diferentes campos del sector de la construcción. Entre los materiales de construcción "modernos" ocupan el primer lugar el cemento y el acero; su abastecimiento es dificultoso. Las consecuencias son, el alza del precio y el incremento de las importaciones, debido a que la capacidad de producción nacional no satisface la demanda, ya que no puede ser ampliada a corto plazo. En casos extremos, se produce una escasez artificial por acaparamiento y por la formación de un floreciente mercado negro; ambos son fenómenos que sólo parcialmente pueden ser controlados por las medidas estatales.

Pero también el mercado de otros materiales de construcción, se pone en movimiento en el remolino de la alta coyuntura. Los productores y comerciantes de materiales de construcción tradicionales, como madera, arena y piedra, conociendo sus posibilidades reaccionan a la nueva situación del mercado con alzas de precio. Un papel decisivo en este proceso, corresponde al encarecimiento de los medios de transporte, debido tanto a causas externas (por ejemplo mayor precio de venta de los fabricantes de camiones extranjeros, devaluación de la moneda nacional con relación a la extranjera) como a causas internas (por ejemplo, mayores ganancias de los importadores, leyes de importación restrictivas).

Nota: En $1977 / 78, \$ 1000.00$ equivalían, aproximadamente, a 37.00 dólares; en 1977 , el salario mínimo vital mensual fijado estatalmente, fue para los obreros de $\$ 1500$, en 1978 de $\$ 1750$. 
Un indicador de esta situación, es el auge de la construcción -reconocido en la investigación económica tradicional como barómetro de la coyuntura- que alcanzó en el Ecuador, en 1976-1977, su máximo valor. En 1978, se registra cierto relajamiento, reflejado en la reducción de los precios de algunos materiales de construcción. En general, los últimos años muestran un alza del nivel de los precios de la construcción de vivienda.

La división de los costos totales de la construcción de vivienda, en costos de material y costos de trabajo, denota el enorme significado de los materiales de construcción como factor de costos. En el programa público de construcción de vivienda MENA I, la proporción de costos de material sobre el total, es del $63.1 \%$; para las ampliaciones y cambios individuales en MENA I, del $68.8 \%$ y para la construcción espontánea de vivienda en ELOÍSA, hasta del 78.1\%. Evidentemente, el significado de los costos de material de construcción se incrementa, a medida que aumenta la informalidad de la construcción, que es la expresión de las posibiliaades de uso flexible de la fuerza de trabajo barata y de la relativamente alta proporción de trabajo no pagado.

\subsection{Problemas en la evaluación}

En el análisis de fenómenos económicos reales, aparecen regularmente problemas de evaluación, cuando se consideran procesos inflacionarios. La determinación de los efectos cuantitativos de la construcción de vivienda presenta este problema. El proceso de construcción es analizado a posteriori. Se estudian, frecuentemente, varios años, habiéndose podido producir durante este lapso diferencias notables en la estructura de precios. La consideración de los efectos indirectos obliga a pensar, de qué modo se debe evaluar el proceso constructivo en relación con los procesos preliminares a la construcción. Además, el mercado de la contrucción es áfectado con especial intensidad por oscilaciones coyunturales y estacionales, de tal manera que es difícil de entender la formación de los precios. Por ejemplo, sucede frecuentemente que los precios de los diferentes materiales de construcción se desarrollan con diferentes tasas inflacionarias. La ubicación de las urbanizaciones, así como las posibilidades de adquisición individuales del propietario-constructor, dificultan también la evaluación del uso de los materiales de construcción. Cuando se hace necesario comparar los efectos de diferentes urbanizaciones y de diferentes tipos de construcción de vivienda, los costos producidos tienen que ser transformados en un común denominador temporal. La construcción de vivienda aparece, por lo tanto, como un sector en el que es necesario considerar en forma especial, las especificaciones espaciales y temporales, para efectuar la evaluación con precios.

Los costos de construcción para el programa estatal de construcción de vivienda MENA I, se refieren a los años 1975/76; los de las construc- 
ciones individuales en MENA I y en ELOÍSA, a los años 1976/77. Los efectos indirectos se basan en datos de los años 1977/78. Para hacer posible la comparación de los efectos producidos por los tres tipos de construcción de vivienda, todos los precios van a ser referidos a 1977/78. Partimos del supuesto que, a pesar de los cambios de los precios, la composición física es constante, o sea, que la función de la producción material de la construcción de vivienda no cambia. Cuando fue posible, los costos de material fueron inflacionados uno por uno. Para los costos de trabajo, se considera un incremento promedio anual, calculado en base a la evolución del salario mínimo vital. Se aceptan las pequeñas variaciones en la división de los costos de construcción totales: en costos de material y de trabajo. Con este proceso de evaluación se elaboran costos calculatorios. La comparación de los efectos se realizó, por lo tanto, sobre la base de los costos que se hubieran producido de haberse llevado a cabo las construcciones en los años 1977/78.

\section{Efectos de ocupación, efectos de ingresos y contenidos de importación} por el uso de materiales de construcción

Los resultados del cálculo de los efectos de ocupación, de los efectos de ingresos y! de los contenidos de importación por el uso de materiales de construcción, en los tres tipos de construcción de vivienda investigados, están reunidos en los cuadros 1, 2 y 3. El resumen de los resultados, que permite la comparación de los efectos, debido al uso de materiales de construcción, se encuentra en el cuadro 4.

Según éstos, la utilización de materiales de construcción por un valor de $\$ 1000$ de $1977 / 78$ produce, en las etapas preliminares de la construcción de la vivienda (en producción y distribución de materiales de construcción), una ocupación de 1.1 a 1.7 días-hombre y un ingreso de $\$ 185$ a \$225. El contenido de importación, es en MENA I, de aproximadamente $4.7 \%$ de los costos de materiales, tanto para las construcciones formales como para las informales; en ELoísA, del $42.5 \%$. En los tres tipos de construcción de vivienda, casi la mitad del valor de los materiales de construcción se debe a importaciones.

Comparando los tres tipos de construcción de vivienda, con relación a los efectos totales de ocupación y de ingresos y de acuerdo con el uso de materiales de construcción, se pueden identificar claramente las siguientes tendencias:

Mientras más informal sea la construcción de vivienda, más ocupación e ingresos produce en las etapas preliminares. El efecto de ocupación en la vivienda informal de ELoísa, es un tercio mayor que el efecto de ocupación por uso de materiales de construcción en la construcción formal de viviendas de MENA I; el efecto de ingresos es un $18 \%$ mayor. Según este dato, con un grado creciente de informalidad en la construcción, el ingreso no se incrementa en igual proporción que la ocupación. 
Cuadro 1

EFECTOS DE OCUPACIÓN, EFECTOS DE INGRESOS Y CONTENIDO DE IMPORTACIÓN POR EL USO DE MATERIALES DE CONSTRUCCIÓN EN EL PROGRAMA ESTATAL DE CONSTRUCCIÓN DE VIVIENDA MENA I

\begin{tabular}{|c|c|c|c|c|c|c|c|c|c|c|c|c|}
\hline \multirow{2}{*}{$\begin{array}{l}\text { Material de } \\
\text { Cons trucciónal }\end{array}$} & \multirow{2}{*}{ Cantidad } & \multirow{2}{*}{$\begin{array}{l}\text { Costos } \\
\text { Material } \\
75 / 76 \\
(1000 \$)\end{array}$} & \multirow{2}{*}{ 告 } & \multirow{2}{*}{$\begin{array}{l}\text { Preciol } \\
\text { unidad } \\
75 / 76 \\
(\$)\end{array}$} & \multirow{2}{*}{$\begin{array}{l}\text { Preciol } \\
\text { unidad } \\
77 / 78 \\
(\$)\end{array}$} & \multirow{2}{*}{$\begin{array}{c}\text { Tasa } \\
\text { de } \\
\text { Inflación } \\
(y)\end{array}$} & \multirow{2}{*}{\multicolumn{2}{|c|}{ 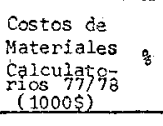 }} & \multicolumn{2}{|c|}{$\begin{array}{l}\text { Efecto de } \\
\text { Ocupacion }\end{array}$} & \multirow{2}{*}{$\begin{array}{l}\text { Efecto } \\
\text { de } \\
\text { Ingresos }\end{array}$} & \multirow{2}{*}{$\begin{array}{l}\text { ontenico } \\
\text { de } \\
\text { impor- } \\
\text { tacion }\end{array}$} \\
\hline & & & & & & & & & $\begin{array}{l}\text { horas } \\
\text { hombre }\end{array}$ & $\begin{array}{c}\text { Ditas I } \\
\text { hombre }\end{array}$ & & \\
\hline (emento naciona $I^{2)}$ & 16215 Saco & 1135 & 2.4 & 70.00 & 75.00 & 21.4 & 1378 & 2.3 & 6201 & 775 & 214 & 298 \\
\hline Cemento importado & $64859 \mathrm{Saco}$ & 5756 & 12.3 & 88.75 & $\$ 1.15 .00$ & 29.6 & 7459 & 12.2 & 17902 & 2238 & 530 & 5848 \\
\hline Acero de cons trucciôn & 141 Tcne ladas & 1719 & 3.7 & 12190 & 10200 & -17.1 & 1424 & 2.3 & 2990 & 374 & 127 & 976 \\
\hline Blogues de concreto & 1692232 Unidad & 9308 & 20.0 & 5.50 & 6.00 & $\begin{array}{r}9.1 \\
5)\end{array}$ & 10154 & 16.6 & 94894 & 12312 & 2152 & 4318 \\
\hline Madera & - & 2751 & 5.9 & - & - & $50^{5)}$ & 4127 & 6.8 & 39207 & 4801 & 887 & 1446 \\
\hline Ventanas de madera & $8270 \curvearrowleft 2$ & 1660 & 3.6 & 200.00 & 360.00 & 80.0 & 2977 & 4.9 & 51800 & 6475 & 867 & 711 \\
\hline Puertas de madera & 3559 Unided & 2758 & 5.9 & 775.00 & 152000 & 96.1 & 5410 & 8.9 & 83855 & 10482 & 1475 & 1893 \\
\hline Vidrio & $8072 \mathrm{~m} 2$ & 969 & 2.1 & 120.00 & 180.00 & 50.0 & 1453 & 2.4 & 4068 & 4068 & 509 & 815 \\
\hline $\operatorname{stras}^{4)}$ & - & 20555 & 44.1 & - & - & $30^{51}$ & 26722 & 43.7 & 236366 & 29347 & 4927 & 12656 \\
\hline & & & & & & & & & 07000 & & 11276 & 896 . \\
\hline
\end{tabular}

Fuente: Cálculos propios.

1 Un día-hombre tiene 8 horas-hombre. $20 \%$ de cemento nacional y $80 \%$ importado.

3 Puertas de madera de $2 \mathrm{~m}^{2}$, incluyendo cerradura.

Los efectos de los otros materiales de construcción fueron calculados de acuerdo a su proporción del total de

los costos de material habiéndose partido del supuesto que producen los mismos efectos que el promedio de materiales de construcción mencionados individualmente.

Ver cuadro 2. 
Cuadro 2

EFECTOS DE OCUPACIÓN, EFECTOS DE INGRESOS Y CONTENIDO DE IMPORTACIÓN POR UTILIZACIÓN DE MATERIALES DE CONSTRUCCIÓN EN LAS MEDIDAS INDIVIDUALES DE CONSTRUCCIÓN DE MENA 1

\begin{tabular}{|c|c|c|c|c|c|c|c|c|c|c|c|c|}
\hline \multirow[t]{2}{*}{$\begin{array}{l}\text { Material de } \\
\text { Construcción }\end{array}$} & \multirow[t]{2}{*}{ Cantidad } & \multirow{2}{*}{$\begin{array}{l}\text { Costos en } \\
\text { material } \\
76 / 777 \\
(\$)\end{array}$} & \multirow[t]{2}{*}{$\%$} & \multirow{2}{*}{$\begin{array}{c}\text { Preciol } \\
\text { Unidad } \\
76 / 77 \\
(\$)\end{array}$} & \multirow{2}{*}{$\begin{array}{c}\text { Preciol } \\
\text { Unidad } \\
77 / 78 \\
(\$)\end{array}$} & \multirow{2}{*}{$\begin{array}{c}\text { Tasa } \\
\text { de } \\
\text { inflaci ôn } \\
(y) \\
\left(\begin{array}{l}q \\
)\end{array}\right.\end{array}$} & \multirow{2}{*}{\multicolumn{2}{|c|}{$\begin{array}{l}\text { Costos de } \\
\text { materiales } \\
\text { cajecuatorios } \\
77 / 78 \\
(\$) \\
\end{array}$}} & \multicolumn{2}{|c|}{$\begin{array}{l}\text { Efecto de } \\
\text { Ocupación }\end{array}$} & \multicolumn{2}{|c|}{$\begin{array}{l}\text { Efecto } \\
\text { de contenide } \\
\text {-Ingresos de }\end{array}$} \\
\hline & & & & & & & & & horibre & hombre $e^{2}$ & & $\begin{array}{l}\text { importa } \\
\text { ción }(S)\end{array}$ \\
\hline Cemento nacional ${ }^{2)}$ & $340 \mathrm{saco}$ & 40920 & 4.1 & 120.35 & 85.00 & -29.4 & 28890 & 2.7 & 150 & 16 & 4481 & 6246 \\
\hline Comento importado & $1360 \mathrm{Saco}$ & 176920 & 17.7 & 130.09 & 115.00 & -11.6 & 156397 & 14.6 & 375 & -47 & 11104 & 122615 \\
\hline Acero de construcción & 6.9 Tone Ladas & 70500 & 7.1 & 10232 & 10100 & -1.3 & 69682 & 6.5 & 146 & 18 & 6195 & 47700 \\
\hline Bloques de concreto & 29250 Unidad & 104340 & 10.4 & 3.57 & $4^{5}$ & 12.0 & 116861 & 10.9 & 1134 & 142 & 24763 & 49701 \\
\hline Ladrillos tradicionales & 24568 Unidad & 47030 & 4.7 & 1.91 & 1.73 & -8.4 & 43079 & 4.0 & 3201 & 400 & 33304 & 2417 \\
\hline Madera & - & 140170 & 14.0 & - & - & $25^{8)}$ & 175213 & 16.4 & 1665 & 208 & 37671 & 61395 \\
\hline Ventanas de madera & $38.7 \mathrm{~m} .2$ & 4560 & 0.5 & 117.83 & $150^{6}$ & 27.3 & 5805 & 0.5 & 101 & 13 & 1692 & 1386 \\
\hline Puertas de madera ${ }^{3}$ & 52 Unidad & 35180 & 3.5 & 576.54 & $\left.800^{6}\right)$ & 18.2 & 41583 & 3.9 & 645 & 81 & 11336 & 1.4550 \\
\hline Ventanas de acero & $67 \mathrm{~m} 2$ & 40000 & 4.0 & $597.01^{\circ}$ & $640^{7}$ & 7.2 & 42880 & 4.0 & 339 & 42 & 7328 & 18254 \\
\hline Puertas de acero & 35 Unidad & 54060 & 5.4 & 1545 & 1630 & 5.5 & 57033 & 5.3 & 308 & 38 & 7511 & 23469 \\
\hline vidrio & $74.5 \mathrm{~m} 2$ & 10805 & 1.1 & 145.03 & 190,00 & 24.1 & 13409 & 1.3 & 38 & 5 & 894 & 7524 \\
\hline $.0 t \operatorname{tros})^{4}$ & & 276300 & 27.6 & - & - & $15^{8)}$ & 317745 & 29.7 & 3414 & 427 & 61799 & 150113 \\
\hline Total & & & & & & 6.8 & 1063577 & 99.8 & 11495 & 1437 & 208073 & 505430 \\
\hline
\end{tabular}

Fúnte: Cálculos propios

1 Un dia-hombre tiene 8 horas-hombre.

2 Se supone una utilización de $20 \%$ de cemento nacional y $80 \%$ cemento importado.

3 Puertas de $2 \mathrm{~m}^{2}$, incluyendo cerradura.
4 Los efectos de los otros materiales de construcción fueron calculados de acuerdo a su proporción del total de los costos de material, habiéndose partido del supuesto que producen los mismos efectos que el promedio de los materiales de construcción mencionados individualmente.

5 Versión ligera de los bloques de concreto; los efectos fueron calculados proporcionalmente a la versión pesada que en $1977 / 78$ costaban $\$ 6$.

- Versión simple.

Versión masiva, en parte con decoraciones de hierro forjado.

8 Según informaciones de comerciantes. 
Cuadro 3

EFECTOS DE OCUPACIÓN, EFECTOS DE INGRESOS Y CONTENIDO DE IMPORTACIÓN POR UTILIZACIÓN DE MATERIALES DE CONSTRUCCIÓN PARA LA CONSTRUCCIÓN DE VIVIENDA DE ELOÍSA

\begin{tabular}{|c|c|c|c|c|c|c|c|c|c|c|c|c|}
\hline $\begin{array}{l}\text { Material de } \\
\text { construcciôn }\end{array}$ & Cantidad & $\begin{array}{l}\text { Costos en } \\
\text { Materia1 } \\
76 / 77 \\
(\$)\end{array}$ & $\frac{2}{3}$ & $\begin{array}{l}\text { Precio/ } \\
\text { Unidad } \\
76 / 77 \\
\text { (\$) }\end{array}$ & $\begin{array}{l}\text { Preciol } \\
\text { Unidad } \\
77 / 78 \\
(\$)\end{array}$ & $\begin{array}{c}\text { Tasa de } \\
\text { Inflaciôn } \\
(y)\end{array}$ & $\begin{array}{c}\text { Costos de } \\
\text { materiales } \\
\text { calculatorios }\end{array}$ & $s$ & $\begin{array}{l}\text { Efec } \\
\text { Ocup } \\
\text { Horas } \\
\text { hombre }\end{array}$ & $\begin{array}{l}\text { cto de } \\
\frac{\text { pación }}{\text { Días }} \\
\text { hombre }\end{array}$ & $\begin{array}{c}\text { Efecto } \\
\text { de } \\
\text { Ingresos } \\
(\$)\end{array}$ & $\begin{array}{c}\text { Contenido } \\
\text { de } \\
\text { Importación } \\
(\$)\end{array}$ \\
\hline $\begin{array}{l}\text { Cemento } \\
\text {.nacional-2f }\end{array}$ & $200 \mathrm{~s} a \cos$ & 22162 & 3.0 & 110.81 & 85.00 & $-23,3$ & 16998 & 2.2 & 76 & 10 & 2636 & 3675 \\
\hline $\begin{array}{l}\text { Cemento } \\
\text { importaco }\end{array}$ & 300 Sacos & 96163 & 13.2 & 120.20 & 115.00 & -4.3 & 92028 & 11.7 & 221 & 23 & 5 334 & 72150 \\
\hline $\begin{array}{l}\text { Acero de } \\
\text { construcción }\end{array}$ & 3.1 Tons. & 32010 & 4.4 & 10326 & 10100 & -2.2 & 32306 & 4.0 & 66 & 8 & 2783 & 21457 \\
\hline $\begin{array}{l}\text { Bloques de } \\
\text { concreto }\end{array}$ & 27030 Unids. & 101004 & 13.9 & 3.74 & $4^{57}$ & 7.0 & 108074 & 13.7 & 1043 & 191 & 22901 & 39140 \\
\hline $\begin{array}{l}\text { Ladrillos } \\
\text { tradicional }\end{array}$ & 25400 unids. & 45850 & 6.3 & 1.81 & 1.75 & -3.3 & 44337 & 5.6 & 3294 & 412 & 34277 & 2487 \\
\hline Madera & - & 121056 & 16.6 & - & - & $25^{8)}$ & 151320 & 19.2 & 1438 & 180 & 32534 & 53023 \\
\hline $\begin{array}{l}\text { Ventanas } \\
\text { de madera }\end{array}$ & $37.3 \mathrm{~m}^{2}$ & 9365 & 1.3 & 248.41 & $250^{6)}$ & 0.6 & 9421 & 1.2 & 164 & 20 & 2746 & 2249 \\
\hline $\begin{array}{l}\text { Puertas } \\
\text { de madera }\end{array}$ & 54 Unids. & 42715 & 8.9 & 791.02 & $300^{6)}$ & 1.1 & 43185 & 5.5 & 669 & 84 & 11772 & 15110 \\
\hline $\begin{array}{l}\text { Ventanas de } \\
\text { acero }\end{array}$ & $36.2 \mathrm{~m}^{2}$. & 21090 & 2.9 & 582.60 & $640^{73}$ & 9.9 & 23178 & 2.9 & 183 & 23 & 3961 & 9867 \\
\hline $\begin{array}{l}\text { Puertas de }{ }^{3)} \\
\text { acero }\end{array}$ & 6 Unids. & 9 910 & 1.4 & 1652 & 1530 & -1.3 & 9781 & 1.2 & 53 & 7 & 1288 & 4025 \\
\hline vidrio & $83.3 \mathrm{~m}^{2}$. & 13450 & 1.8 & 161.46 & 180.00 & $\begin{array}{r}11.5 \\
8)\end{array}$ & 14997 & 1.9 & 42 & 5 & 1000 & 8415 \\
\hline otros ${ }^{4)}$ & - & $212 \quad 377$ & 29.2 & - & - & $15^{8)}$ & $244 \quad 234$ & 31.0 & 3259 & 407 & 55005 & $104 \quad 051$ \\
\hline Tota & & $727 \quad 152$ & 99.9 & & & 8.5 & 788859 & 100.1 & क 513 & 1315 & $17743: 3$ & 395649 \\
\hline
\end{tabular}

Fuente: Cálculos propios.

1 Un día-hombre tiene 8 horas-hombre.

2 Se supone una utilización de $20 \%$ cemento nacional y $80 \%$ cemento importado.

3 Puertas de $2 \mathrm{~m}^{2}$, incluyendo cerradura.

4 Los efectos de los otros materiales de construcción fueron calculados de acuerdo a su proporción del total de los costos en material, habiéndose partido del supuesto que producen los mismos efectos que el promedio de los materiales de construcción mencionados individualmente.

Versión ligera.

7 Versión masiva.

8 Según comerciantes. 
Un indicador de esto, son los sueldos y salarios pagados para la producción y distribución de materiales de construcción, los ingresos por díahombre (ver cuadro 4). Evidentemente, en la construcción informal se utilizan materiales cuya producción y distribución generan menores ingresos por día-hombre, que los materiales referidos en la construcción formal de vivienda.

\section{Cuadro 4}

COMPARACIÓN DE LOS EFECTOS POR USO DE MATERIALES DE CONSTRUCCIÓN

\begin{tabular}{|c|c|c|c|c|}
\hline \multirow{2}{*}{$\begin{array}{l}\text { Urbanización } \\
\text { Tipos de construccion } \\
\text { de vivienda }\end{array}$} & \multicolumn{3}{|c|}{$\begin{array}{c}\text { Por } \$ 1000 \text { en costos calculatorios en thaterial } \\
\qquad 1977 / 78\end{array}$} & \multirow{2}{*}{$\begin{array}{c}\text { Ingresos por } \\
\text { dia-hombre } \\
\text { (\$) }\end{array}$} \\
\hline & $\begin{array}{l}\text { Efectos de ocupaciôn } \\
\text { (dias-hombre) }\end{array}$ & $\begin{array}{l}\text { Efecto de Ingresos } \\
(\$)\end{array}$ & $\begin{array}{l}\text { Contenido de } \\
\text { importación } \\
\text { (\$) }\end{array}$ & \\
\hline MENA I formaI & 1.11 & 184.54 & 473.96 & 166.25 \\
\hline MENA I informal & 1.34 & 194.72 & 472.99 & 145.31 \\
\hline ELOISA informal & 1.67 & 224.93 & 425.94 & 134.69 \\
\hline
\end{tabular}

Fuente: Cuadros 1,2 y 3 y cálculos propios.

Los distintos tipos de construcción de vivienda se diferencian, sustancialmente, según la composición de los materiales usados. De acuerdo con esto, cada material de construcción tiene un valor proporcional diferente en el total de los costos de material.

A continuación se muestra, cómo el uso de determinados materiales de construcción, tiene una influencia diferente en los efectos totales, en cada tipo de construcción de vivienda; o sea, en qué medida, los efectos dependen del uso de materiales diferenciales según el tipo de construcción. La meta de estas consideraciones, es elaborar una clasificación por tipos de materiales de construcción de acuerdo con la influencia que tienen sobre los efectos investigados. Los resultados están reunidos en los cuadros 5,6 y 7 .

El cuadro 5 muestra la participación porcentual de los diferentes materiales de construcción en los efectos totales, en cada tipo de construcción de vivienda. En los tres tipos de construcción se usan, por ejemplo, bloques de concreto, pero con distinta intensidad, como lo indican las diferentes proporciones sobre el total de los costos de material. El aporte de los bloques de concreto a los efectos de ocupación y de ingresos totales, debido a la utilización de materiales de construcción, en MENA I formal, está por debajo de la proporción de los costos de material en ELoísA, debido a que allí se han usado también ladrillos tradicionales, que tienen mayor efectividad sobre la ocupación y los ingresos que los bloques de concreto.

El contenido de importación de las ventanas y puertas de acero corresponde en EloísA, exactamente a los costos de material; en las cons- 
trucciones individuales, en MENA 1 , se encuentra por debajo, debido a que evidentemente aquí fueron usados materiales de construcción con un mayor contenido de importación que en ELoísa. En forma similar también otros materiales de construcción tienen una influencia variable sobre los efectos, en los tres tipos de construcción de vivienda.

Para dar una visión general de la gran cantidad de materiales de construcción, éstos fueron agrupados en tres rubros. El criterio para esta división, fue la similitud de la influencia sobre los efectos investigados. EI primer grupo de materiales de construcción es poco efectivo sobre la ocupación y los ingresos y tiene un alto contenido de importación. En este grupo se encuentran reunidos el cemento importado, acero de construcción y vidrio. En el segundo grupo están el cemento nacional, bloques de concreto, puertas y ventanas de acero. Estos materiales ocasionan medianos efectos de ocupación, ingresos y contenidos de importación. En el tercer grupo figuran ladrillos tradicionales, madera, ventanas y puertas de madera, que se caracterizan por altos efectos de ocupación y de ingresos, con un bajo contenido de importación.

La participación de estos grupos de materiales en los costos totales de material, para los tres tipos de construcción de vivienda, y su aporte a cada tipo de efecto total, están representados en el cuadro 6. Con relación al uso diferencial según el grado de informalidad de la construcción, es posible identificar, sólo en el caso del tercer grupo, una clara tcndencia: mientras más informal es la construcción, mayor es la proporción de materiales de construcción tradicionales. La posibilidad de comparar está limitada considerablemente por la diferente proporción de "otros materiales de construcción".

Relativizando la influencia del rubro "otros materiales de construcción" sobre los efectos, la participación porcentual de los grupos de materiales de construcción se relacionó con la parte que representaban dentro de los costos de material. Se elaboraron coeficientes de efectividad que indican, en qué proporción un grupo de materiales de construcción tiene efecto sobre los totales del respectivo tipo de construcción de vivienda, cuando participa en un $1 \%$ del total de los costos de material. El cuadro 7 contiene los coeficientes de efectividad en cifras porcentuales. La tabla de los coeficientes de efectividad es un instrumento con el que puede ser estimada en forma cuantitativa, la influencia de los diferentes materiales de construcción sobre la ocupación, los ingresos y el contenido de importación en los diferentes tipos de construcción.

El grupo I muestra la siguiente característica: con informalidad creciente en la construcción, los efectos de ocupación y de ingresos disminuyen; por el contrario, el contenido de importación se incrementa. Mientras que en la construcción de vivienda informal de MENA I, los materiales de construcción del grupo II todavía producen efectos de ocupación y de ingresos sobre el promedio, éstos bajan relativamente en las construcciones informales y están por debajo del promedio. La influencia sobre el 
Cuadro 5

Participación de los diferentes Materiales de CONSTRUCCIÓN SOBRE LOS EFEctos pOR tipos DE CONSTRUCCIÓN DE VIVIENDA

(partes porcentuales)

\begin{tabular}{|c|c|c|c|c|c|c|c|c|c|c|c|c|}
\hline \multirow[b]{2}{*}{$\begin{array}{c}\text { Material } \\
\text { de } \\
\text { Construcción }\end{array}$} & \multicolumn{4}{|c|}{ MENA I formal } & \multicolumn{4}{|c|}{ MENA I informal } & \multicolumn{4}{|c|}{ ELOISA informal } \\
\hline & $\begin{array}{l}\text { Costos } \\
\text { do } \\
\text { Material } \\
1977 / 78\end{array}$ & $\begin{array}{l}\text { Efecto } \\
\text { de } \\
\text { ocupacion }\end{array}$ & $\begin{array}{l}\text { Efecto } \\
\text { de } \\
\text { Ingresos }\end{array}$ & $\begin{array}{l}\text { Contenido } \\
\text { de } \\
\text { Inportación }\end{array}$ & $\begin{array}{l}\text { Costos } \\
\text { \& } \\
\text { Haterial } \\
1977 / 78\end{array}$ & $\begin{array}{l}\text { Efecto } \\
\text { de } \\
\text { Ocupacibn }\end{array}$ & $\begin{array}{l}\text { Efecto } \\
\text { de } \\
\text { Ingresos }\end{array}$ & $\begin{array}{c}\text { Contenido } \\
\text { de } \\
\text { Importaci5n }\end{array}$ & $\begin{array}{l}\text { Costos } \\
\text { en } \\
\text { Material } \\
1977 / 78\end{array}$ & $\begin{array}{c}\text { Efecto } \\
\text { de } \\
\text { Ocupacisa }\end{array}$ & $\begin{array}{l}\text { Efecto } \\
\text { de } \\
\text { Ingresos }\end{array}$ & $\begin{array}{l}\text { Contenido } \\
\text { de } \\
\text { Importacisn }\end{array}$ \\
\hline $\begin{array}{l}\text { Cemento } \\
\text { nacional }\end{array}$ & 2.3 & 1.1 & 1.9 & 1.0 & 2.7 & 1.1 & 2.2 & 1.2 & 2.2 & 0.8 & 1.5 & 1.1 \\
\hline $\begin{array}{l}\text { Cemento } \\
\text { importado }\end{array}$ & 12.2 & 3.3 & 4.7 & 20.2 & 14.6 & 3.3 & 5.3 & 24.3 & 11.7 & 2.1 & 3.7 & 21.5 \\
\hline $\begin{array}{l}\text { Acero de } \\
\text { construcción }\end{array}$ & $5^{2.3}$ & 0.6 & 1.1 & 3.4 & 6.5 & 1.3 & 3.0 & 9.4 & 4.0 & 0.6 & 1.6 & 6.4 \\
\hline $\begin{array}{l}\text { Bloques de } \\
\text { concreto }\end{array}$ & 16.6 & 18.2 & 19.1 & 14.9 & 10.9 & 9.9 & 11.9 & 9.8 & 13.7 & 10.0 & 12.9 & 11.7 \\
\hline $\begin{array}{l}\text { Ladrillos } \\
\text { tradicionales }\end{array}$ & es & - & - & - & 4.0 & 27.8 & 16.0 & 0.5 & 5.6 & 31.3 & 19.3 & 0.7 \\
\hline Madera & 6.8 & 7.2 & 7.9 & 5.0 & 16.4 & 14.5 & 18.1 & 12.1 & 19.2 & 13.7 & 18.3 & 15.8 \\
\hline $\begin{array}{l}\text { Ventanas de } \\
\text { madera }\end{array}$ & $\cdot 4.9$ & 9.6 & 7.7 & 2.5 & 0.5 & 0.9 & 0.8 & 0.3 & 1.2 & 1.5 & 1.5 & 0.7 \\
\hline $\begin{array}{l}\text { Puertas de } \\
\text { madera }\end{array}$ & 8.9 & 15.5 & 13.1 & 6.5 & 3.9 & 5.5 & 5.4 & 2.9 & 5.5 & 6.4 & 6.6 & 4.5 \\
\hline $\begin{array}{l}\text { Ventanas de } \\
\text { acero }\end{array}$ & - & - & - & - & 4.0 & 2.9 & 3.5 & 3.6 & 2.9 & 1.7 & 2.2 & 2.9 \\
\hline $\begin{array}{l}\text { Puertas de } \\
\text { acero }\end{array}$ & - & - & - & - & 5.3 & 2.6 & 3.6 & 4.6 & 1.2 & 0.5 & 1.2 & 0.7 \\
\hline Vidrio & 2.4 & 0.8 & 0.9 & 2.8 & 1.3 & 0.3 & 0.4 & 1.5 & 1.9 & -.4 & 0.6 & 2.5 \\
\hline Otros & 43.7 & 43.7 & 43.7 & 43.7 & 29.7 & 29.7 & 29.7 & 29.7 & 31.0 & 31.0 & 31.0 & 31.0 \\
\hline$T \circ t a 1$ & 100.1 & 100.0 & 100.0 & 100.0 & 99.8 & 99.9 & 99.9 & 99.9 & 100.1 & 100.0 & 99.9 & 100.0 \\
\hline
\end{tabular}

Fuente: Cuadros 1, 2 y 3, cálculos propios. 


\section{Cuadro 6}

PaRTicipación de los gRUPOS DE MATERIALES DE CONSTRUCCIÓN SOBRE LOS EFECTOS POR TIPOS DE CONSTRUCCIÓN DE VIVIENDA

(partes porcentuales)

\begin{tabular}{|c|c|c|c|c|c|c|c|c|c|c|c|c|}
\hline \multirow[b]{2}{*}{$\begin{array}{c}\text { Material } \\
\text { de } \\
\text { Construcciôn }\end{array}$} & \multicolumn{4}{|c|}{ MEHA I formaI } & \multicolumn{4}{|c|}{ MENA I informE 1} & \multicolumn{4}{|c|}{ ELOISA informal } \\
\hline & $\begin{array}{c}\text { Costos } \\
\text { us } \\
\text { Material }\end{array}$ & $\begin{array}{l}\text { Efecto } \\
\text { de } \\
\text { Ocupación }\end{array}$ & $\begin{array}{c}\text { Efecto } \\
\text { de } \\
\text { Ingresos }\end{array}$ & $\begin{array}{c}\text { Contenido } \\
d a \\
\text { Importaciót }\end{array}$ & $\begin{array}{l}\text { Costos } \\
0 \\
\text { Material }\end{array}$ & $\begin{array}{l}\text { Efecto } \\
\text { de } \\
\text { decupacion }\end{array}$ & $\begin{array}{c}\text { Efecto } \\
\text { de } \\
\text { Ingresos }\end{array}$ & $\begin{array}{l}\text { Contenido } \\
\text { de } \\
\text { Importación }\end{array}$ & $\begin{array}{l}\text { Costos } \\
\text { ans } \\
\text { Material }\end{array}$ & $\begin{array}{c}\text { Efecto } \\
\text { de } \\
\text { de }\end{array}$ & $\begin{array}{c}\text { Efecto } \\
\text { de } \\
\text { Ingresos }\end{array}$ & $\begin{array}{l}\text { Contenido } \\
\text { on } \\
\text { Importacisn }\end{array}$ \\
\hline $\begin{array}{l}\text { Cemento im- } \\
\text { portado } \\
\text { Acero de } \\
\text { construcción } \\
\text { yidrio }\end{array}$ & 16.9 & 4.7 & 6.7 & 26.4 & 22.4 & 4.9 & 8.7 & 35.2 & 17.6 & 3.1 & 5.9 & 30.4 \\
\hline $\begin{array}{l}\text { Femento na- } \\
\text { cional } \\
\text { Blogues de } \\
\text { concreto } \\
\text { Yentanas } y \\
\text { puertas de } \\
\text { acero }\end{array}$ & 18.9 & 19.3 & 21.0 & 15.9 & 22.3 & 15.5 & 21.2 & 19.2 & 20.0 & 13.0 & 17.3 & 16.9 \\
\hline $\begin{array}{l}\text { Ladrillos tra- } \\
\text { dicionales } \\
\text { Madera } \\
\text { Puertas y ven- } \\
\text { tanas de ma- } \\
\text { dera }\end{array}$ & - & 32.3 & 28.6 & 14.0 & 24.8 & 48.8 & 40.3 & 15.8 & 31.5 & 52.9 & 45.7 & 21.7 \\
\hline Otros & 43,7 & 43.7 & 43.7 & 43.7 & 29.7 & 28.7 & 29.7 & 29.7 & $\$ 1.0$ & 31.0 & 31.0 & $\$ 1.0$ \\
\hline Total & 100.1 & 100.0 & 100.0 & 100.0 & 99.8 & 99.9 & 99.3 & 99.9 & 100.1 & 100.0 & 100.0 & 100.0 \\
\hline
\end{tabular}

Fuente: Cuadro 5, cálculos propios. 


\section{Cuadro 7}

COEFICIENTES DE EFECTIVIDAD DE LOS GRUPOS DE MATERIALES DE CONSTRUCCIÓN POR TIPO DE CONSTRUCCIÓN DE VIVIENDA (EN PORCENTAJE Y REPRESENTACIÓN GRÁFICA)

\begin{tabular}{|c|c|c|c|c|c|c|c|c|c|c|}
\hline & \multirow{2}{*}{$\begin{array}{l}\text { Grupo de material } \\
\text { de construccion }\end{array}$} & \multicolumn{3}{|c|}{ UENA I formal } & \multicolumn{3}{|c|}{ MENA I informa 1} & \multicolumn{3}{|c|}{ ELorSA informal } \\
\hline & & $\begin{array}{l}\text { Efecto } \\
\text { de } \\
\text { Ocupación }\end{array}$ & $\begin{array}{l}\text { Efecto } \\
\text { de } \\
\text { Ingresos }\end{array}$ & $\begin{array}{c}\text { Contenido } \\
\text { de } \\
\text { Importación }\end{array}$ & $\begin{array}{l}\text { Efecto } \\
\text { de } \\
\text { Ocupaciôn }\end{array}$ & $\begin{array}{l}\text { Efecto } \\
\text { de } \\
\text { Ingresos }\end{array}$ & $\begin{array}{l}\text { Contenido } \\
\text { de } \\
\text { Import ación }\end{array}$ & $\begin{array}{l}\text { Efecto } \\
d e \\
\text { Ocupación }\end{array}$ & $\begin{array}{l}\text { Efecto } \\
\text { da } \\
\text { Ingresos }\end{array}$ & $\begin{array}{c}\text { Contenido } \\
\text { de } \\
\text { Inportación }\end{array}$ \\
\hline I & $\begin{array}{l}\text { Cemento importado } \\
\text { Acero de construccion } \\
\text { vidrio }\end{array}$ & 0.28 & 0.40 & 1.56 & 0.22 & 0.39 & 1.57 & 0.18 & 0.34 & 1.73 \\
\hline II & $\begin{array}{l}\text { Cemento nacionai } \\
\text { Bloques de concreto } \\
\text { Ventanas y puertas de acero }\end{array}$ & 1.02 & 1.11 & 0.84 & 0.72 & 0.93 & 0.84 & 0.65 & 0.87 & 0.85 \\
\hline III & $\begin{array}{l}\text { Ladrillos tradicionales } \\
\text { Madera }\end{array}$ & 1.57 & 1.39 & 0.68 & 1.97 & 1.63 & 0.64 & 1.58 & 1.45 & 0.63 \\
\hline
\end{tabular}

Fuente: Cuadro 6, cálculos propios. 
contenido de importación no se diferencia en los tres tipos de construcción. El contenido de importación de los materiales de construcción del grupo III es de acuerdo con la definición, inferior al contenido de importación de los otros materiales de construcción. Las diferencias según los tipos de construcción no son significativas. Sin embargo, comparativamente, los coeficientes de efectividad de los materiales de construcción de este grupo, son elevados en relación a la ocupación y los ingresos, en la construcción informal de MENA I, lo cual es, principalmente, consecuencia del uso de ladrillos tradicionales, que en el programa de vivienda de MENA I no habían sido utilizados. En ELoísA, por el contrario, en total, se utilizan más materiales tradicionales, de modo que los efectos de ocupación y de ingresos relacionados con los costos de material, en promedio, están por debajo de los valores de MENA I.

\section{Ejemplo de aplicación: sustitución de materiales de construcción}

Con el coeficiente de efectividad de los grupos de materiales de construcción, se puede estimar la influencia de materiales alternativos de diferentes grupos sobre la ocupación, los ingresos y el contenido de importación. La tabla de los coeficientes de efectividad da respuesta a la pregunta: ¿Qué efecto se produciría sobre los sectores preliminares de la construcción de vivienda, si un material de un grupo se sustituyera por otro de otro grupo? Para esto se parte de los supuestos siguientes: 1) la composición de los costos de material permanece constante, 2) los materiales son de hecho sustituibles y, 3) los materiales alternativos están disponibles al mismo precio. Estas condiciones van a ser satisfechas en mayor grado cuando menores sean los costos de los materiales a sustituir. Según las investigaciones existentes se ofrecen tres posibilidades de sustitución: cemento importado por cemento nacional, bloques de concreto por ladrillos tradicionales y, puertas y ventanas de acero por puertas y ventanas de madera.

\section{a) Sustitución de bloques de concreto por ladrillos tradicionales en MENA I formal}

La sustitución de un material de construcción del grupo II por uno del grupo III origina, para un monto determinado de costos de material, en MENA I formal, los siguientes cambios en las etapas preliminares de construcción de la vivienda: se genera un $53.9 \%$ más de ocupación y un $25.2 \%$ más de ingreso; el contenido de importación se reduce en un $23.5 \%$. La sustitución de bloques de concreto por ladrillos tradicionales en MENA I formal produce, según las cifras del cuadro 1, un incremento de la ocupación de 6336 días-hombre, un incremento del ingreso de $\$ 542000$ y un ahorro de divisas de $\$ 1015000$. 
b) Sustitución de cemento importado por cemento nacional en MENA I informal

Si se sustituye un material de construcción del grupo I -en MENA I informal - por un material de construcción del grupo II, manteniendo constante la suma de los costos de material, se producen los siguientes cambios en la producción y distribución de materiales de construcción: el efecto de ocupación se incrementa en $227.3 \%$, el efecto de ingresos en $138.5 \%$; el contenido de importación se reduce un $46.5 \%$. Utilizando las cifras del cuadro 2, la sustitución de cemento importado por nacional produce 107 días-hombre más en ocupación, y $\$ 15379$ más en ingresos; el contenido de importación se reduciría, debido a esta medida, en \$57016.

c) Sustitución de ventanas y puertas de acero por ventanas y puertas de madera en ELOÍsA

Ventanas y puertas de acero pertenecen al grupo II; ventanas y puertas de madera al grupo III. Colocando en ELoísa ventanas y puertas de madera, en vez de ventanas y puertas de acero, se obtendrían, manteniendo constante la suma de los costos de material, los siguientes cambios, en las etapas preliminares de construcción de la vivienda: la ocupación se incrementaría en $158.5 \%$, el ingreso en $66.7 \%$, el contenido de importación se reduciría en $18.8 \%$. La sustitución de ventanas y puertas de acero, por ventanas y puertas de madera, tendría, utilizando las cifras del cuadro 3, las siguientes consecuencias: el efecto de ocupación se incrementaría en 48 días-hombre, el efecto de ingresos en $\$ 3501$ y el contenido de importación disminuiría en \$2 612 .

\section{Efectos de ocupación y de ingresos directos $e$ indirectos}

Debido a que los efectos indirectos, por uso de materiales de construcción, fueron obtenidos sobre la base de datos de los años 1977/78, y los de efectos directos provienen del período 1975/1977, estos últimos deben ser extrapolados con ayuda de las tasas de cambio de los precios de 1977/78, con el fin de permitir la unificación de ambos. En el cuadro 8, se inflacionaron los costos de construcción totales; los costos efectivos se transformaron en costos calculatorios de 1977/78. La división de los costos totales de construcción en costos de material y costos de trabajo, sufrió un cambio mínimo al inflacionarla.

La división de los costos en costos de materiales y de trabajo, demuestra cómo, con informalidad creciente en la construcción, la proporción de los costos de material se incrementa. En el programa estatal de construcción de vivienda MENA $\mathrm{I}$, el $36.1 \%$ del total de los costos de construcción, corresponde a costos de trabajo; en la construcción indivi- 
dual de MENA I, el $32.4 \%$ y en la construcción de vivienda informal de ELoísa sólo el $22.7 \%$. Dado que en la construcción de vivienda formal, en promedio, no se pagan sueldos más altos que en la informal, la reducida parte de los costos de trabajo en la construcción informal de vivienda, se puede explicar sólo por una alta proporción de trabajo no pagado.

En el cuadro 9, se presentan los efectos de ocupación y de ingresos directos e indirectos, de los tres tipos de construcción de vivienda. Como referencia, se utilizó para el total de las construcciones investigadas, una suma de los costos de construcción del orden de los $\$ 1000$, y junto con la división porcentual, también la superficie techada de un metro cuadrado.

Para esta última dimensión de referencia, no se pudieron obtener datos comparables para el caso de MENA I informal. Con la construcción individual de MENA I, no sólo se produjo un nuevo espacio habitable, sino que se modificó y mejoró el espacio habitable existente del programa estatal, de tal manera que los costos y efectos no se pueden adjudicar unívocamente a la producción de superficie techada. Los datos de MENA I informal, referidos a la producción de un metro cuadrado de superficie techada, contienen por lo tanto, una proporción no definible con exactitud, de modo que serán puestos entre paréntesis.

Considerando un valor total de $\$ 1000$, se produce en la construcción formal de viviendas de MENA I, una ocupación total de 3.9 días-hombre; en las construcciones informales de MENA I, 4.3 días-hombre, y en la construcción informal de vivienda de ELoísA, 6.1 días-hombre. Con informalidad ascendente de la construcción, no sólo se incrementa el efecto de ocupación total, también crecen ambos componentes: el efecto de ocut pación directo y el indirecto. Para la construcción de un metro cuadrado de superficie techada se necesita, en la construcción informal de vivienda, una menor ocupación que en la formal. En ELofsA, el efecto total de ocupación es de 4.8 días-hombre por metro cuadrado de superficie techada, en MENA I formal, es de 6.4 días-hombre.

El efecto de ocupación total se divide, en el programa estatal de construcción de vivienda MENA I, en $82.1 \%$ de ocupación en la obra y $17.9 \%$ de ocupación en los sectores preliminares de la producción de materiales de construcción y en su distribución. En MENA I informal, las partes correspondientes son: el $79.1 \%$ y $20.9 \%$ y en ELoísA de $78.9 \%$ y $21.1 \%$. Mientras más informal es la construcción, mayor es el incremento de la parte de los efectos de ocupación indirectos. Diez puestos de trabajo en la obra de construcción formal, corresponden aproximadamente, a dos puestos de trabajo en los sectores preliminares; en el informal a casi tres. La construcción informal de vivienda está, según estos datos, interrelacionada en mayor grado con los sectores preliminares, cuando se considera al mismo tiempo la ocupación.

Debido a que la construcción formal de viviendas MENA I, ocasiona un gasto de $\$ 1000$, se produce un efecto de ingresos total de $\$ 453$ : $\$ 335$ 
Cuadro 8

Costos efectivos y costos calculatorios $1977 / 78$

\begin{tabular}{|c|c|c|c|c|c|c|}
\hline $\begin{array}{l}\text { Urbanización } \\
\text { ripo de } \\
\text { construcción } \\
\text { de vivienda } \\
\end{array}$ & Tipos de costo & $\begin{array}{l}\cos \text { tos } \\
\text { efectivos } \\
(\$ \hat{y})^{1}\end{array}$ & $\%$ & $\begin{array}{c}\text { Tasa de } \\
\text { Inffacion } \\
(\xi)\end{array}$ & $\begin{array}{c}\text { Costos } \\
\text { calculatorios } \\
(\$ 11)\end{array}$ & $\%$ \\
\hline \multirow{3}{*}{$\begin{array}{l}\text { HENA I } \\
\text { formal }\end{array}$} & costos en material & 46611 & 63.1 & 31.1 & 61104 & 63.9 \\
\hline & Costos en trabajo & 27261 & 36.9 & $26.7^{2)}$ & 34540 & 36.1 \\
\hline & Costos totales & 73872 & 100.0 & 29.5 & 95644 & 100.0 \\
\hline \multirow{3}{*}{$\begin{array}{l}\text { KENA I } \\
\text { informal }\end{array}$} & Costos en material & 1000885 & 68.8 & 6.8 & 1068945 & 67.6 \\
\hline & Costos en trabajo & 452870 & 31.2 & $13.3^{2)}$ & 513102 & 32.4 \\
\hline & Costos totales & 1453755 & 100.0 & 8.8 & 1582047 & 100.0 \\
\hline \multirow{3}{*}{$\begin{array}{c}\text { ELOISA } \\
\text { informal }\end{array}$} & Costos en material & 727152 & 78.1 & 8.5 & 788859 & 77.3 \\
\hline & Costos en trabajo & $201+140$ & 21.9 & $13.3^{2)}$ & 231291 & 22.7 \\
\hline & Cestos totales & 331291 & 100.0 & 9.5 & 1020150 & 100.0 \\
\hline
\end{tabular}

Fuente: Cuadros 1, 2 y 3; cálculos propios.

1 Costos de MENA I formal en $\$ 1000$.

Para la inflacionación de los costos en trabajo se supuso un incremento anual en salario de $13.33 \%$, para los cálculos se parte del "Salario mínimo vital"; es en 1975 de $\$ 1250$, en 1978 de $\$ 1750$. 
Cuadro 9

EFECTOS DE OCUPACIÓN Y DE INGRESOS DIRECTOS E INDIRECTOS

\begin{tabular}{|c|c|c|c|c|c|}
\hline $\begin{array}{l}\text { Urbanizacibn } \\
\text { Tipo de construccion } \\
\text { de vivienda }\end{array}$ & $E f a c t a s$ & $\begin{array}{l}\text { Costos } \\
\text { totales } \\
(\$)^{1)}\end{array}$ & $\begin{array}{c}\text { Por } \$ 1 \text { ooo de } \\
\text { costo total de } \\
\text { la construeción } \\
\text { (\$) }\end{array}$ & $\begin{array}{l}\text { Por } m^{2} \text { da } \\
\text { superficie } \\
\text { techada } \\
(\$)\end{array}$ & $\%$ \\
\hline \multirow{5}{*}{$\begin{array}{l}\text { MENA } T \\
\text { formaI }\end{array}$} & Efecto de ingresos directo & 32019 & 334.77 & 542.27 & 33.5 \\
\hline & Efecto de ingresos indirecto & 11276 & 117.90 & 190.97 & 11.8 \\
\hline & Residuo & 23388 & 244.53 & 396.10 & 24.5 \\
\hline & Contenido de importacibn & 28961 & 302.80 & 490.48 & 30.3 \\
\hline & costo total de la construccion & 95644 & $\pm 000,00$ & 1619.82 & 100.1 \\
\hline \multirow{5}{*}{$\begin{array}{l}\text { MENA I } \\
\text { informal }\end{array}$} & Efecto de ingresos directo & 513102 & 324.33 & 532.82 & 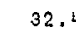 \\
\hline & Efecto de ingresos indirecto & 208078 & 131.52 & 216.07 & 13.2 \\
\hline & Residuo & $355 \quad 437$ & 224.67 & 369.09 & 22.5 \\
\hline & Contenido de importacion & 505430 & 319.48 & 524.85 & 31.9 \\
\hline & Costo total de la construccion & 1582047 & 1000.00 & 1642.83 & 100.0 \\
\hline \multirow{5}{*}{$\begin{array}{l}\text { ELOISA } \\
\text { informá }\end{array}$} & Efecto de ingresos directo & 231291 & 226.72 & 179.55 & 22. \\
\hline & Efecto de ingresos indirecto & 177438 & 173.93 & 137.74 & 17.4 \\
\hline & Residuo & 275772 & 270.32 & 214.07 & 27.0 \\
\hline & Contenido de importacion & 335649 & 329.02 & 260.56 & 32.5 \\
\hline & Costo total de 1 a construccion & 1020150 & 1000.00 & 791.92 & 100. \\
\hline
\end{tabular}

Fuente: Cuadros $1,2,3$ y 8 , cálculos propios

1 Datos para las medidas de construcción totales en $\$ 1000$.

Para el efecto de ingresos directo sólo se consideraron ingresos netos; son en 1975/76 7.36 por debajo del ingreso bruto; se ha supuesto esta tasa para el cálculo de ingreso neto calculatorio de 1977/78.

4 Incluso trabajo no pagado (64.9\% del total del trabajo realizado). 
corresponden a los participantes directos del proceso constructivo en forma de sueldos y salarios, $\$ 118$ a los ingresos en la producción y distribución de materiales de construcción. Si bien en las construcciones individuales MENA I, el efecto de ingresos directo es menor, el indirecto es mayor, de tal manera que el efecto de ingresos total es algo más alto que en MENA I formal. En la construcción de vivienda informal de ELoísA, el efecto de ingresos total referido a $\$ 1000$ en costos de construcción es de $\$ 401$; se compone de una parte directa de $\$ 227$ y una indirecta de $\$ 174$.

Con mayor claridad que en los casos anteriores, la comparación de los efectos de ingresos, muestra mayor interrelación en la construcción de vivienda de los sectores preliminares, cuando la informalidad de la construcción es creciente. En la construcción formal de vivienda de MENA I, el $26 \%$ del total de los ingresos es producto de la producción y distribución de materiales de construcción; en MENA I informal el 29\%, y en la construcción de vivienda informal de eloísa hasta el $43 \%$. Por $\$ 100$ pagados por la construcción de vivienda a los trabajadores de la construcción, los destinados a los sectores preliminares son $\$ 35$ en el caso de la construcción formal de vivienda MENA I, y $\$ 77$ en el caso de la construcción informal de vivienda en ELoísA.

Por metro cuadrado de superficie techada, el efecto total de ingresos en MENA I formal, es más del doble del que se produce en ELof́sa; el efecto de ingresos directos asciende al triple. Esto demuestra la importancia de la parte de auto-ayuda en la construcción de vivienda informal. De hecho, aproximadamente el $65 \%$ del trabajo realizado en ELoísA no fue pagado. En razón de la auto-ayuda, los costos de trabajo pudieron ser reducidos por lo menos a la mitad y se ahorró aproximadamente el $20 \%$ del total de los costos de la construcción. En las construcciones individuales de modificación y ampliación de MENA I, la proporción de auto-ayuda es de aproximadamente el $28 \%$ del total del trabajo realizado, o sea mucho menos que la construcción informal de ELoísa. Por auto-ayuda, los costos de trabajo de MENA I informal fueron reducidos aproximadamente en un cuarto y el total de los costos de la producción se redujo aproximadamente en un $13 \%$.

Resumiendo, haciendo la comparación entre los efectos de ocupación y de ingresos directos e indirectos según el tipo de construcción de vivienda, se destacan las siguientes tendencias:

- Con informalidad creciente de la contrucción, se incrementan los efectos de ocupación, al mantenerse una suma constante de costos de construcción; los efectos de ingreso directos disminuyen cuando aumenta la proporción de auto-ayuda.

- La realización de una superficie techada de igual dimensión, exige en la construcción de vivienda formal, considerablemente mayor ocupación que en la construcción informal; en la construcción formal, sobre todo, los efectos de ingresos directos se incrementan sobreproporcionalniente, en relación a los efectos de ocupación; esto también es una con- 
secuencia de la alta proporción de trabajo no pagado en la construcción informal de vivienda.

- Los efectos directos producidos en la obra son parcialmente superiores a los efectos indirectos por producción y distribución de materiales de construcción; mientras más informales las actividades de construcción, mayor será la proporción de efectos indirectos. La construcción informal de vivienda está, por lo tanto, más intensamente relacionada con los sectores preliminares que la construcción formal de vivienda.

\section{Efectos de ingresos y contenidos de importación}

Partimos del argumento de que el valor de cada producto - así como el de la vivienda - puede ser dividido, por una parte, en ingresos nacionales y por otra, en importación. El ingreso producido en la obra y el ingreso por producción y distribución de materiales de construcción, fueron identificados como efectos de ingresos directos e indirectos en el capítulo anterior, así como los contenidos de importación incorporados en los materiales de construcción lo fueron en el cap. 2. La suma restante que complementa las partes mencionadas para dar el total de los costos de construcción, se denomina en el cuadro 10 "residuo". Forman el residuo, tanto los ingresos generados en etapas de producción y distribución anteriores a lo ya investigado, como también las ganancias de la empresa no comprendidas en los efectos de ingresos indirectos.

Los costos totales de construcción por metro cuadrado de superficie techada, extrapolados en el período $1977 / 78$, en el programa estatal de construcción de vivienda MENA I, son de $\$ 1620$, más del doble que en la construcción informal de vivienda en eloísa, donde son de $\$ 792$. Ya se indicó el ahorro de costos por auto-ayuda en la construcción informal de vivienda. Otro motivo para los mayores costos de la construcción formal de vivienda, está en el uso de materiales de construcción más caros. Por ejemplo en MENA I, para la edificación de paredes, se usaron sólo bloques de concreto, mientras que en ELoísa también se utilizaron los considerablemente más baratos ladrillos tradicionales. Pero, por sobre todo, el nivel más alto, es decir, el mejor equipamiento de la infraestructura interna, debe haber producido los costos más altos por metro cuadrado, en la construcción formal de vivienda, especialmente las instalaciones sanitarias y eléctricas.

Con relación a los efectos de ingresos directos e indirectos, la comparación de las tres medidas de construcción investigadas, confirma las mismas tendencias que se perfilan en el cap. 3. Mientras más informal es la construcción, con mayor intensidad desciende el efecto de ingreso directo, como parte del total de los costos de construcción. En la construcción formal de vivienda de MENA I, el 33.5\% del total de los costos de construcción corresponde a ingresos directos; en MENA I informal, el 
Cuadro 10

EFECTO DE INGRESOS Y CONTENIDOS DE IMPORTACIÓN

\begin{tabular}{|c|c|c|c|c|c|c|}
\hline $\begin{array}{l}\text { Urbanizacion } \\
\text { Tipo de construceiör } \\
\text { de vivienda }\end{array}$ & $E \neq c t o$ & $s$ & $\begin{array}{l}\text { Costos } \\
\text { Totales }\end{array}$ & $\begin{array}{l}\text { Por } \$ 1000 \text { de } \\
\text { costo total de } \\
\text { la construccion }\end{array}$ & $\begin{array}{l}\text { Por } \pi^{2} \cdot \text { de } \\
\text { superficie } \\
\text { techada }\end{array}$ & $\%$ \\
\hline \multirow{6}{*}{$\begin{array}{l}\text { MENA I } \\
\text { EOrma }\end{array}$} & \multirow{3}{*}{$\begin{array}{l}\text { Efecto de Ocupación } \\
\text { (dias-hombre) }\end{array}$} & Directo & 309276 & 3.23 & 5.24 & 82.1 \\
\hline & & Indirecto & 67613 & 0.71 & 1.15 & 17.9 \\
\hline & & rotal & 376889 & 3.94 & 6.39 & 100.0 \\
\hline & \multirow{3}{*}{$\begin{array}{l}\text { Efecto de Ingresos } \\
\text { (Sucres) }\end{array}$} & Directo ${ }^{2)}$ & 32019 & 334.77 & 542.27 & 74.0 \\
\hline & & Indinecto & $1127 \hat{~}$ & 117.90 & 190.97 & 25.0 \\
\hline & & Total & 43295 & 452.6 .7 & 733.24 & 100.0 \\
\hline \multirow{7}{*}{$\begin{array}{l}\text { MENA I } \\
\text { informal }\end{array}$} & \multirow{3}{*}{$\begin{array}{c}\text { Efecto de ocupacion } \\
\text { (dias-hombra) }\end{array}$} & Directo ${ }^{3)}$ & 5429 & 3.43 & 5.64 & 79.1 \\
\hline & & Indirecto & 1437 & 0.91 & 1.49 & 20.9 \\
\hline & & Iotal & 6866 & 4.34 & 7.13 & 100.0 \\
\hline & & & & & & \\
\hline & \multirow{3}{*}{$\begin{array}{l}\text { Efecto de Ingresos } \\
\text { (Sucres) }\end{array}$} & Directo & $513 \quad 102$ & 324.33 & 532.81 & 71.1 \\
\hline & & Indirecto & $208 \quad 078$ & 131.52 & 216.07 & 28.9 \\
\hline & & Total & 721180 & 455.85 & 748.89 & 100.0 \\
\hline \multirow{6}{*}{$\begin{array}{l}\text { ILOISA } \\
\text { informal }\end{array}$} & \multirow{3}{*}{$\begin{array}{c}\text { Efecto de ocupación } \\
\text { (dias-hombre) }\end{array}$} & Directo ${ }^{4)}$ & 4919 & 4.82 & 3.82 & 78.9 \\
\hline & & Indirecto & 1315 & 1.29 & 1.02 & 21.1 \\
\hline & & Total & 6234 & 6.11 & 4.84 & 100.0 \\
\hline & \multirow{3}{*}{$\begin{array}{l}\text { Efecto de Ingresos } \\
\text { (Sucres) }\end{array}$} & Directo & 231291 & 229.72 & 179.55 & 56.6 \\
\hline & & Indirecto & 177438 & 173.93 & 137.74 & 43.4 \\
\hline & & Total & 408729 & 400.85 & 317.29 & 100.0 \\
\hline
\end{tabular}

Fuente: Cuadros $1,2,8$ y 9 , cálculos propios.

1 Datos para MENA I formal en $\$ 1000$. 
$32.4 \%$ y en la construcción informal de vivienda ELoísA, solamente el $22.4 \%$. Paralelamente crece la proporción de efectos de ingresos indirectos de $11.8 \%$ en MENA I formal, hasta $17.4 \%$ en ELOísA.

La proporción del residuo sobre el total de los costos de construcción, muestra que no hay una tendencia unívoca dependiente del tipo de construcción. Este hecho se debe a que, en esta suma restante, están reunidos diferentes tipos de ingresos en diferentes etapas preliminares. La alta proporción del residuo sobre el total de los costos en ELoísA, puede ser interpretado, con cuidado, como consecuencia de que la construcción informal de vivienda está relacionada con mayor intensidad que la formal, con sectores anteriores a la producción y distribución de materiales de construcción. Esta explicación parece aceptable, si se considera que la construcción informal está relacionada también con la producción y distribución de materiales de construcción más intensamente que la formal.

La proporción relativamente alta del residuo en relación con los costos de la construcción informal de vivienda, da lugar a otra suposición, que por falta de información no puede ser aún documentada. Es de suponer que los sectores preliminares a la construcción de vivienda, tengan mayores ganancias, cuando abastecen a la construcción informal que cuando aprovisionan a la formal. La sobre-demanda de materiales de construcción anima al comercio a explotar sus posibilidades de ganancia con la construcción informal de vivienda, ya que ésta no es controlada.

Los contenidos de importación representan, en la construcción formal de vivienda MENA I, el 30.3\%; en la construcción individual de MENA I el $31.9 \%$ y en la construcción informal de vivienda de ELoísa el $32.9 \%$ del total de los costos de construcción. Según estos datos, el contenido de importación repercute con mayor intensidad en la construcción informal de vivienda que en la construcción formal. Sin embargo, no se verifican diferencias importantes en los diferentes tipos de construcción.

Resumiendo, se puede decir que casi la mitad del costo de materiales o casi un tercio del total de los costos de construcción, fluye fuera del país (en forma de divisas) para la importación de materiales de construcción y servicios preliminares para la producción y distribución de materiales de construcción. 